\title{
Relativistic Fe K $\alpha$ line and ensemble spins of black holes in narrow-line Seyfert 1 galaxies
}

\author{
Weimin Yuan, Zhu Liu, Youjun Lu and Xin-Lin Zhou
}

National Astronomical Observatories, Chinese Academy of Sciences, Beijing 100012, China

email: wmy@nao.cas.cn

\begin{abstract}
While a broad line of the Fe K $\alpha$ emission is commonly found in the X-ray spectra of typical Seyfert galaxies, the situation is unclear in the case of Narrow Line Seyfert 1 galaxies (NLS1s) - an extreme subset which are generally thought to harbor less massive black holes with higher accretion rates. We report results of our study of the assemble property of the $\mathrm{Fe}$ $\mathrm{K}$ line in NLS1s by stacking the X-ray spectra of a large sample of 51 NLS1s observed with XMM-Newton. We find in the stacked X-ray spectra a prominent, broad emission feature over 4-7 keV, which is characteristic of the broad $\mathrm{Fe} \mathrm{K} \alpha$ line. Our results suggest that a relativistic broad Fe line may in fact be common in NLS1s. The line profile is used to study the average spin of the black holes in the sample. We find, for the first time, that their black holes are constrained to be likely spinning at averagely low or moderate rates as a population. The implications of the results are discussed in the context of the black hole growth in NLS1 galaxies.
\end{abstract}

Keywords. line: profile, galaxies: Seyfert, X-rays: galaxies

\section{Introduction}

A broad emission line at $E \sim 6-7 \mathrm{keV}$ has long been found in the X-ray spectra of about $\sim 40 \%$ of Seyfert 1 galaxies (e.g., Nandra et al. 2007). It is generally interpreted to be the Fe $\mathrm{K} \alpha$ emission produced via the K-shell process in the proximity of supermassive black holes and is hence relativistically broadened (Fabian et al. 1989). The energy of the red wing of the broad line is determined by the inner radius of the accretion disc, which is commonly believed to be at the innermost stable circular orbit (ISCO). The ISCO is strongly dependent on the the black hole spin. As such, the profile of a relativistic broad Fe $\mathrm{K} \alpha$ line can be used to constrain the spin of the black hole (Brenneman \& Reynolds 2006; Dauser et al. 2010). In observations, black hole spins have been measured in about two dozen individual bright AGNs (e.g., Patrick et al. 2012; Walton et al. 2013). However, for the vast majority of AGNs it is impossible to constrain the black hole spin with this method, which requires a very high signal-to-noise ratio of the X-ray spectra at the Fe K $\alpha$ energies (de La Calle Pérez et al. 2010). Alternatively, spectral stacking is an effective way to obtain a composite spectrum with very high signal-to-noise for a certain class of objects, e.g., Corral et al. (2008); Iwasawa et al. (2012)

Narrow Line Seyfert 1 galaxies (NLS1s) are a subset of active galactic nuclei (AGNs) (Osterbrock \& Pogge 1985; Goodrich 1989), which are generally thought to have relatively small black hole masses (Boroson 2002) and higher accretion rates close to the Eddington rate (Boroson \& Green 1992). They show extreme properties among AGNs (Komossa 2008). Concerning the broad Fe K $\alpha$ line in NLS1s, somewhat contradictory results have been presented in the literature. A few well-studied NLS1s show apparently a broad Fe K $\alpha$ emission line, including SWIFT J2127.4+5654 (Miniutti et al. 2009; Marinucci et al. 2014) and 1H 0707-495 (Fabian et al. 2009). de La Calle Pérez et al. (2010) found that the broad Fe $\mathrm{K} \alpha$ lines were detected in 4 out of 30 NLS1s in their full sample and in 
2 out of 4 in their flux limited sample. Ai et al. (2011) found evidence for significant Fe $\mathrm{K} \alpha$ lines in none of the objects in a sample of 13 extreme NLS1s that have very small broad-line widths $\left(<1200 \mathrm{~km} \mathrm{~s}^{-1}\right)$. It is not known whether a broad Fe $\mathrm{K} \alpha$ line is common in NLS1s. We investigate the ensemble property of the Fe K $\alpha$ line of NLS1s by stacking the X-ray spectra of a large sample of NLS1s observed with XMM-Newton. We use the cosmological parameters $H_{0}=70 \mathrm{~km} \mathrm{~s}^{-1} \mathrm{Mpc}^{-1}, \Omega_{\mathrm{M}}=0.27$ and $\Omega_{\Lambda}=0.73$. All quoted errors correspond to the 90 per cent confidence level unless specified otherwise

\section{Sample}

Two NLS1s catalogues were used to compiled a sample of NLS1s that have XMMNewton observations. The first is the largest NLS1s sample $(\sim 2000)$, which is homogeneously selected from the SDSS DR3 by Zhou et al. (2006) with well measured optical spectrometric parameters. The second includes about 400 NLS1s from the AGN catalogue compiled by Véron-Cetty \& Véron (2006). Only sources with more than 100 net source counts in either the EPIC PN or the combined MOS detectors in the $2-10 \mathrm{keV}$ band were selected. We excluded three objects that are previously known to show a significant broad Fe K $\alpha$ line, namely, $1 \mathrm{H}$ 0707-495, Mrk 766, and NGC 4051. The sample includes 51 NLS1s that have a total of 68 observations.

The X-ray spectra are retrieved from the LEDAS website†. We combined the MOS1 and MOS2 spectra if both are available. The PN and the combined MOS spectra are treated independently. No significant broad $\mathrm{Fe} \mathrm{K} \alpha$ line is found in individual spectra which is likely due to relatively low $\mathrm{S} / \mathrm{N}$ in the Fe line region.

\section{Stacked X-ray spectrum of NLS1s}

A method introduced by Corral et al. (2008) was adopted to stack the X-ray spectra of objects with different redshifts from different observations. We outline the main procedures here and refer readers to the original paper for details and discussions about the method. We first fit $2-10 \mathrm{keV}$ spectra with an absorbed power-law model, ignoring 5-7 keV energy range. Then we reconstruct the source spectra by unfolding the observed spectra. The unfolded spectra are rescaled and de-redshifted to the source rest frame. Finally, the stacked spectrum is obtained by rebinning and averaging the rescaled and de-redshifted spectra.

Figure 1 (left-hand panel, blue points with error bars) shows the stacked $2-10 \mathrm{keV}$ spectrum. A prominent broad emission feature in the $5-7 \mathrm{keV}$ energy range superposing the power-law continuum is clearly shown. We test the reliability of the presence of this feature, by means of Monte Carlo simulations.

Using simulations similar to those in Corral et al. (2008), we check whether the broad emission feature is an artifact produced by the stacking procedure. For each source, we simulate 100 power-law plus absorption continuum spectra and produce 100 stacked continuum spectra to construct the confidence intervals for the underlying continuum of the composite source spectrum. Figure 1 (left-hand panel; open circles) shows the composite simulated spectrum as well as the 68 per cent $(1 \sigma)$ and 95 per cent $(2 \sigma)$ confidence intervals. It can be seen that the broad line feature of the composite source spectrum in the $\mathrm{Fe} \mathrm{K} \alpha$ region fall at or out of the $2 \sigma$ confidence levels. We also found that the method has almost no affect on a broad line profile, as in our case here. However, narrow lines remain unresolved in the unfolded spectra with a width comparable to the

$\dagger$ http://www.ledas.ac.uk/ 

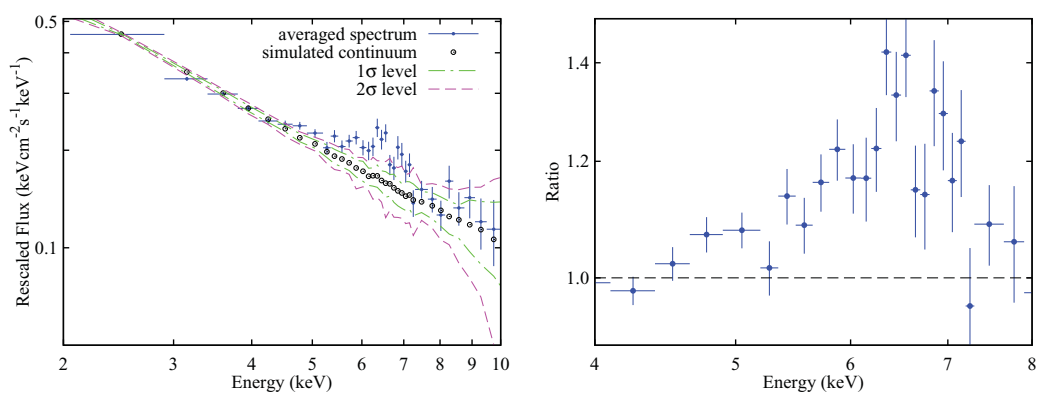

Figure 1. Left-hand panel: Stacked source spectrum (blue points with error bars), simulated power-law continuum (open circles), and its confidence ranges. The green dot-dashed line and the red dashed line are the $1 \sigma$ and $2 \sigma$ levels for each bin, respectively. Right-hand panel: Ratio of the stacked source spectrum to the stacked simulated power-law continuum in the $4-8 \mathrm{keV}$ range. Figures from Liu et al. (2015)
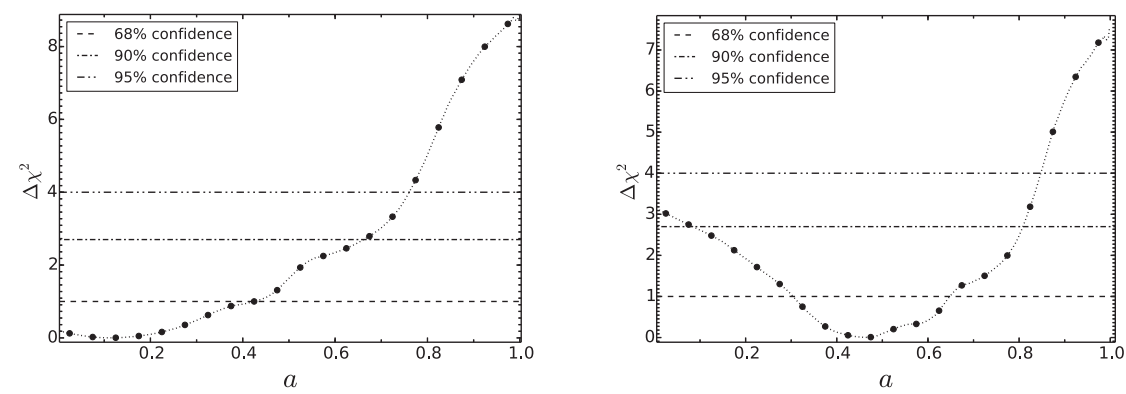

Figure 2. Error contour for the spin parameter, calculated using the RELLINE model. Left-hand Panel: Case A (with a $6.97 \mathrm{keV}$ narrow emission line); Right-hand Panel: Case B (with a $6.67 \mathrm{keV}$ narrow absorption line). Figures from Liu et al. (2015)

intrinsic instrumental energy resolution, which is energy dependent (Corral et al. 2008; Iwasawa et al. 2012). We thus conclude that the broad emission feature in $5-7 \mathrm{keV}$ in the composite source spectrum should be real.

\section{Broad Fe line and black hole spin}

The ratio of the stacked source spectrum to the stacked simulated power-law continuum is shown in the right-hand panel in Figure 1. It's clearly shown a broad line profile. In addition, there is an indication of a high ionization emission $(6.97 \mathrm{keV}) /$ absorption $(6.67 \mathrm{keV}) \mathrm{Fe} \mathrm{K}$ line. Hereafter, we referred emission line scenario as Case A and absorption line scenario as Case B. We fit the composite spectrum with broad Fe K $\alpha$ line

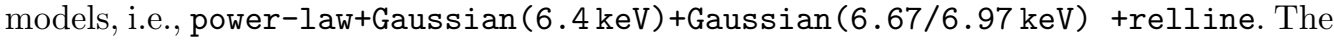
line energy of the highly ionized Fe $\mathrm{K}$ line is fixed at $6.97 \mathrm{keV}$ or $6.67 \mathrm{keV}$ with line width of $85 \mathrm{eV}$ for Case A and Case B, respectively. Only the broad line energy and the spin of $\mathrm{BH}$ are free parameters in the relline model with emissivity index fixed at 3.0 and the disk inclination fixed at 30 degree. This model leads an acceptable fit $\left(\chi^{2} /\right.$ d.o.f $=23 / 21$ for both Case A and B). In both cases, the spin can only be well constrained if we fixed the photon index to that obtained from the stacked simulated power-law spectra: in case A $a<0.66$ (90 per cent significance level, see Figure 2, left panel) with a best-fit value of 0.10 and in case B $a=0.47_{-0.39}^{+0.34}$ (90 per cent significance level, Figure 2, right panel). The inferred black hole spin is not very high in either case. 


\section{Discussion}

Our results indicate that the bulk of the black holes of NLS1s in the nearby universe (a median redshift 0.085 ) either have averagely low or moderate spins, or distribute in a wide range of spins, from low spins to high ones. This is the first time that the ensemble black hole spin parameters are constrained for NLS1s as a population. In Case A, the spin $a<0.66$ may suggest that the black holes in NLS1s may grow via mainly the process of chaotic accretion, which lead to low values of the final average spins, e.g., $a=0.1-0.3$ as found by King et al. (2008). In Case B, the black hole spin parameter is constrained to be widely distributed $(a<0.81)$. If this is ture, it suggest that the growth of each of these black holes is due to chaotic accretion with many episodes, in each episode the accretion is prolonged with a significant increase of black hole mass and significant spin evolution.

\section{Summary}

In this work, we study the ensemble property of the Fe K $\alpha$ line in NLS1s by stacking the X-ray spectra. A broad line feature is found in the composite spectrum of NLS1s and can be well fitted with a relativistic Fe line. Our results suggest that a relativistic Fe line may be common in NLS1s. There are tentative indications for low or intermediate spin of BHs in NLS1. This may imply that the BHs in NLS1s may grow mainly via chaotic accretion if the spin is low. Otherwise, if the spin is intermediate and widely distributed, the growth of BHs should be due to chaotic accretion with many episodes, the accretion is prolonged in each episode.

\section{References}

Ai Y. L., Yuan W., Zhou H. Y., Wang T. G., \& Zhang S. H., 2011, ApJ, 727, 31

Boroson T. A., 2002, ApJ, 565, 78

Boroson T. A. \& Green R. F., 1992, ApJS, 80, 109

Brenneman L. W. \& Reynolds C. S., 2006, ApJ, 652, 1028

Corral A., et al. 2008, A\& $A, 492,71$

Dauser T., Wilms J., Reynolds C. S., \& Brenneman L. W., 2008, MNRAS, 409, 1534

de La Calle Pérez I., et al. 2010, A\&A A, 524, A50

Fabian A. C., Rees M. J., Stella L., \& White N. E., 1989, MNRAS, 238, 729

Fabian A. C., et al. 2009, Nature, 459, 540

Goodrich R. W., 1989, ApJ, 342, 224

Iwasawa K., et al. 2012, A\& $A, 537, \mathrm{~A} 86$

King A. R., Pringle J. E., \& Hofmann J. A., 2008, MNRAS, 385, 1621

Komossa S., 2008, in Rev. Mex. Astron. Astrofis. Conf. Ser. Vol. 32, p. 86

Marinucci A., et al. 2014, MNRAS, 440, 2347

Liu Z., Yuan W., Lu Y., \& Zhou X. L., 2015, MNRAS, 447, 517

Miller J. M., 2007, ARA $\& A, 45,441$

Miniutti G., et al., 2009, MNRAS, 398, 255

Nandra K., O'Neill P. M., George I. M., \& Reeves J. N., 2007, MNRAS, 382, 194

Osterbrock D. E. \& Pogge R. W., 1985, ApJ, 297, 166

Patrick A. R., et al. 2012, MNRAS, 426, 2522

Tanaka Y., et al. 1995, Nature, 375, 659

Turner T. J., et al. 2002, ApJL, 574, L123

Véron-Cetty M.-P., \& Véron P., 2006, A\&̊A, 455, 773

Walton D. J., Nardini E., Fabian A. C., Gallo L. C., \& Reis, R. C. 2013, MNRAS, 428, 2901

Zhou H., Wang T., Yuan W., Lu H., Dong X., Wang J., \& Lu Y., 2006, ApJS, 166, 128 